\title{
Palatovaginal (pharyngeal) artery: clinical implication and surgical experience
}

\author{
Apostolos Karligkiotis · Luca Volpi • Vincenzo Abbate • \\ Paolo Battaglia · Francesco Meloni • Mario Turri-Zanoni • \\ Maurizio Bignami $\cdot$ Paolo Castelnuovo
}

Received: 27 February 2014 / Accepted: 19 May 2014

(C) Springer-Verlag Berlin Heidelberg 2014

\begin{abstract}
The palatovaginal or pharyngeal artery is a small branch of the internal maxillary artery supplying the nasopharynx. Bleeding from this artery is exceptional and only one case of traumatic epistaxis from this artery has been reported previously. We report a case of a 66-year-old male presenting with right recurrent posterior epistaxis. Endoscopic dissection of the pterygopalatine fossa and direct visualization of the palatosphenoidal canal permitted to identify the origin of bleeding, and coagulation of the pharyngeal artery solved the epistaxis. Although rare, intractable posterior epistaxis may arise from the pharyngeal artery. The anatomical knowledge of this artery and of the palatosphenoidal canal is of outmost importance in endoscopic transpterygoid and nasopharyngeal procedures, to identify the vidian canal, evaluate nasopharyngeal cancer spread in the pterygopalatine fossa, reduce bleeding during surgery of the nasopharynx, and harvest adequately the pedicle of the nasoseptal flap.
\end{abstract}

Keywords Palatovaginal artery · Vidian canal . Pharyngeal artery $\cdot$ Palatosphenoidal canal $\cdot$ Endoscopic sinus surgery $\cdot$ Skull base $\cdot$ Pterygopalatine fossa $\cdot$ Internal maxillary artery $\cdot$ Sphenopalatine artery

A. Karligkiotis $(\bowtie) \cdot$ L. Volpi · F. Meloni

Division of Otorhinolaryngology, Department of Surgical, Microsurgical and Medical Sciences, University of Sassari, Azienda Ospedaliero Universitaria, Viale San Pietro 43, 07100 Sassari, Italy

e-mail: alkis.karligkiotis@gmail.com

\section{Abbate}

Department of Maxillofacial Surgery, University of Naples

"Federico II", Naples, Italy

P. Battaglia $\cdot$ M. Turri-Zanoni · M. Bignami · P. Castelnuovo Division of Otorhinolaryngology, Department of Biotechnology and Life Sciences, University of Insubria, Varese, Italy

\section{Introduction}

The palatovaginal artery (PVA) [or more correctly known as the pharyngeal artery $(\mathrm{PhA})]$ is a minute branch that arises from the internal maxillary artery (IMA) in the pterygopalatine fossa (PPF) [1]. The PVA and canal of the same name are rarely mentioned and even omitted in the medical literature describing the anatomy of the PPF. The PVA exits through the posterior bound of the PPF, entering in the palatovaginal canal (PVC). This canal is a bone tunnel formed by the upper edge of the sphenoid process of the palatine bone and the anteroinferior wall of the sphenoid sinus (Fig. 1), located infero-medially to the vidian canal. The PVC courses medially from the edge of the medial pterygoid plate to the posterior part of the roof of the nasopharynx [2]. Since the palatine bone does not articulate with the vaginal process of the sphenoid bone, PinheiroNeto et al. [2] in their anatomical study, suggested that the term "palatovaginal" is a misnomer and the canal containing the PhA should be named palatosphenoidal canal (PSC). Once in the canal, the artery courses posteriorly and emerges from the PSC in the nasopharynx (Fig. 2) to enter in an area of rich anastomoses in the region of the Eustachian tube [3].

The aim of this article is to report a case of spontaneous epistaxis from the PhA treated surgically and present the difficulties in the diagnosis and management of this rare condition. The importance and role of the PhA in advanced endoscopic skull base procedures are also discussed.

\section{Case report}

A 66-year-old male was referred to our department for a right side epistaxis. The patient was hemodynamically stable, 


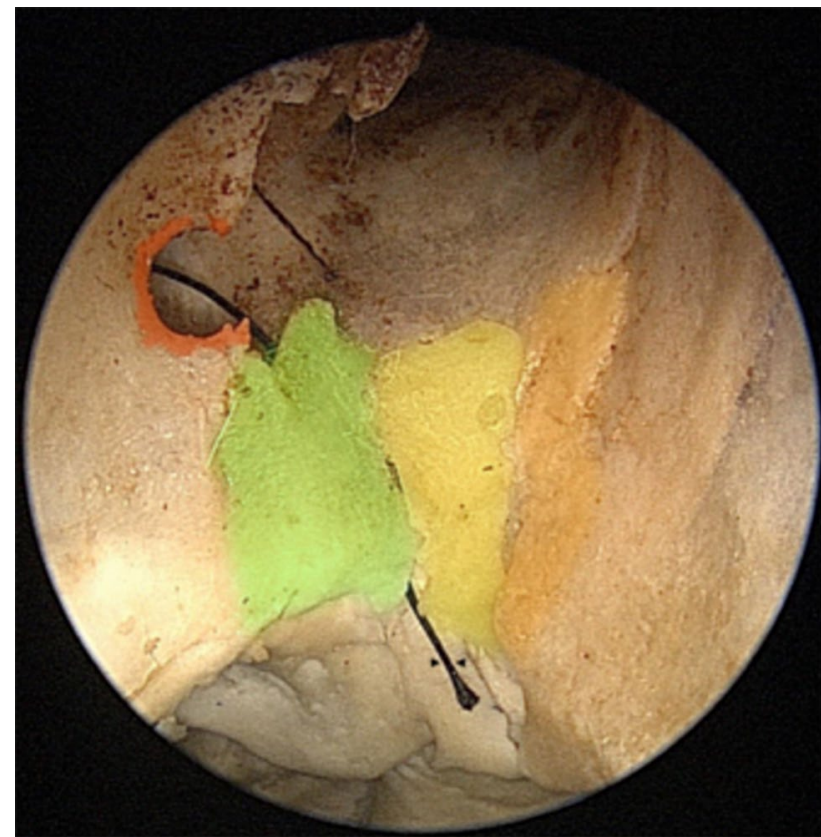

Fig. 1 Endoscopic view of the osteology of the palatosphenoidal canal (PSC) in a dry skull. The PSC is formed by the upper edge of the sphenoid process of the palatine bone and the anteroinferior wall of the sphenoid sinus. A black thread is passing through the right PSC. Red sphenopalatine foramen, green sphenoid process of the palatine bone, yellow basisphenoid, orange vomer

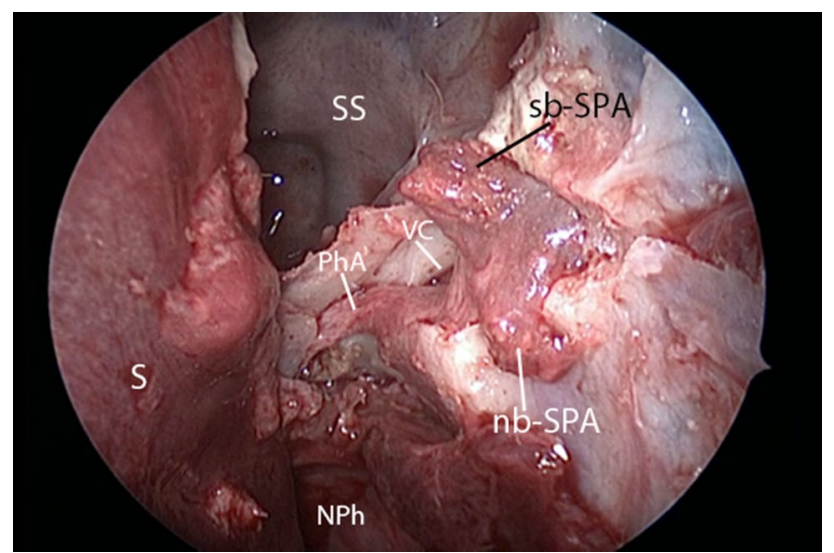

Fig. 2 Endoscopic dissection of the left pterygopalatine fossa (PPF) in a cadaveric specimen. The sphenoid process of the palatine bone has been removed to expose the pharyngeal artery $(\mathrm{PhA})$, originating from the PPF and reaching the mucosa of the nasopharynx (NPh), through the palatosphenoidal canal. $n b-S P A$ nasal branches of the sphenopalatine artery, $s b-S P A$ septal branches of the sphenopalatine artery, $S$ septum, $S S$ sphenoid sinus, $V C$ vidian canal

with a blood pressure of 130/80 mmHg, normal blood count, coagulation screening and biochemistry exams. A right nasal packing was performed with temporary control of the bleeding. After 2 days, the packing was removed but persistent bleeding was noticed from the right sphenopalatine region in

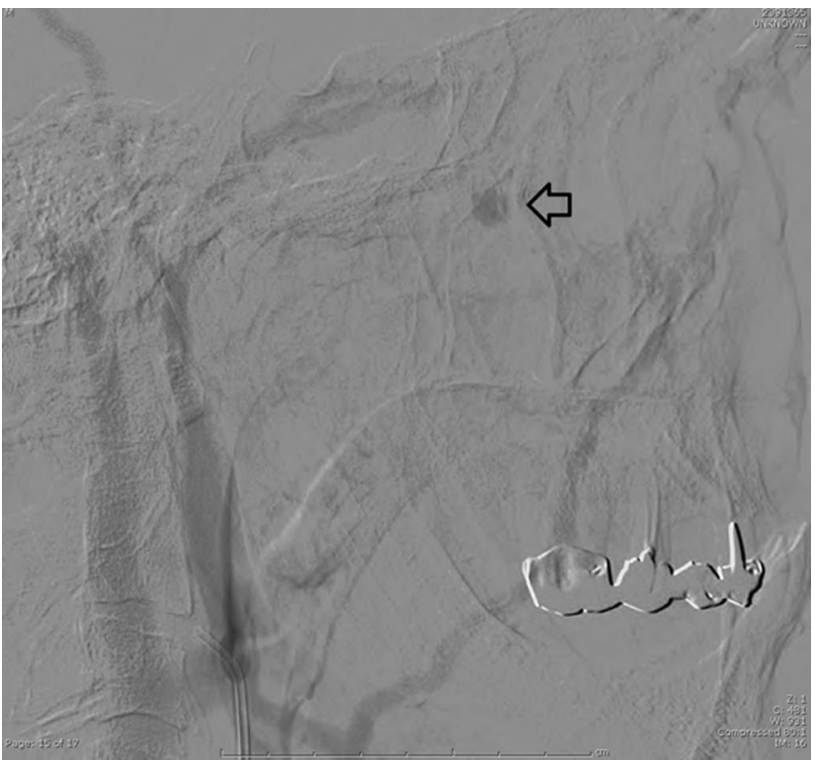

Fig. 3 Right-sided transfemoral selective angiography of the external carotid artery (ECA), in local anesthesia, targeted to investigate bleeding points in the sinonasal cavity. Slight evidence of tardive postcontrast mucosal spreading (black arrow) from the distal branches of internal maxillary artery (IMA), in the anterior region of the basisphenoid, is evident after contrast injection of the ECA

the endoscopic examination. The patient was packed again and subsequently underwent bipolar coagulation of the right sphenopalatine artery (SPA) and its branches under general anesthesia, with apparent control of the bleeding.

After 15 days, the patient presented again to our department with a new episode of severe right epistaxis associated to anemia due to the persistent bleeding ( $\mathrm{Hb} 8.0 \mathrm{~g} / \mathrm{dl})$. He underwent a selective angiography of the epiaortic vessels with evidence of bleeding from the area of the distal branches of the right IMA with tardive postcontrast mucosal spreading at the level of the basisphenoid (Fig. 3). At the same time, distal embolization of the right IMA was performed with embospheres $(300-500 \mu \mathrm{m})$. However, after the procedure, no resolution of the bleeding was obtained.

The patient was then submitted to a second endoscopic procedure. Once a wide medial antrostomy associated to spheno-ethmoidectomy was performed, the orbital process of the palatine bone and the medial portion of the posterior wall of the maxillary sinus were removed to expose the PPF. An accurate dissection of the branches of the pterygopalatine portion of IMA was performed. Removing of the sphenoid process of the palatine bone exposed the bleeding point coming from the $\mathrm{PhA}$, which was then coagulated (Fig. 4) and sectioned. At the end of the procedure, three units of red blood cells were transfused and the patient was discharged after 3 days with stable $\mathrm{Hb}$ levels $(11.5 \mathrm{~g} / \mathrm{dl})$. At 3 years of follow-up, no new episodes of epistaxis were observed. 


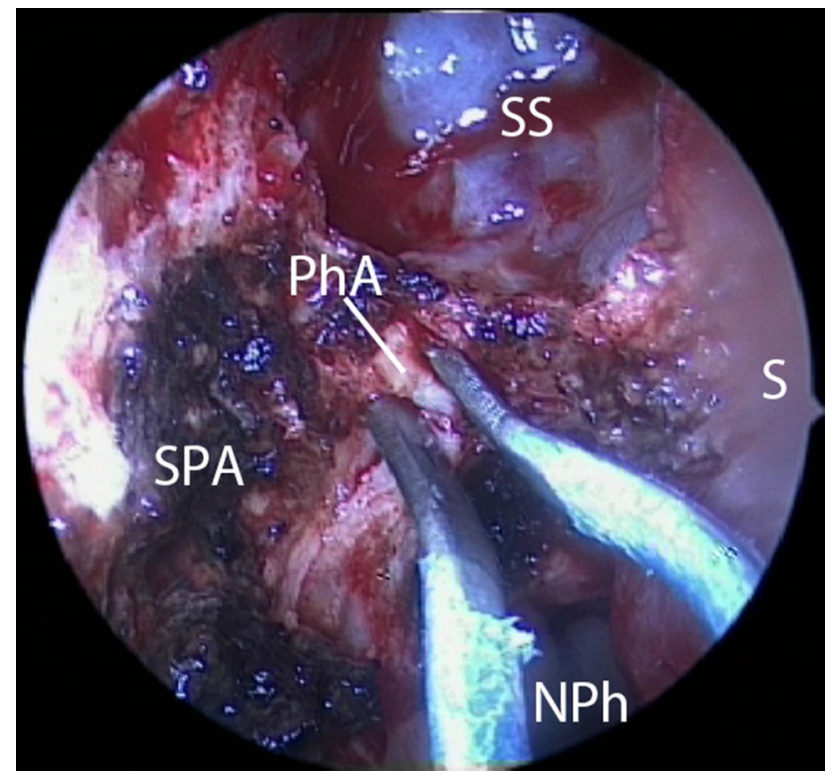

Fig. 4 Intraoperative endoscopic view with $0^{\circ}$ endoscope during bipolar coagulation of the right pharyngeal artery $(\mathrm{PhA})$ at the time of the second surgical procedure. The sphenoid process of the palatine bone has been removed and the PhA has been exposed. The previous coagulation of the sphenopalatine artery (SPA) can be observed. NPh nasopharynx, $S$ septum, $S S$ sphenoid sinus

\section{Discussion}

Intractable posterior epistaxis predominantly arises from the SPA; however, other branches of the pterygopalatine portion of IMA, such as the descending palatine artery (DPA) and $\mathrm{PhA}$, may also be the origin of posterior nasal bleeding [3].

Otolaryngologist not familiar with the PhA may misdiagnose this rare but possible point of origin of epistaxis. Only one case of significant intractable epistaxis from the PhA developing after traumatic injury of the PSC and treated with embolization of the distal IMA has been described in the medical literature [3]. In our case, initial coagulation of the SPA and subsequent embolization of the distal IMA were not able to stop the bleeding. Only after endoscopic dissection of the pterygopalatine fossa and direct visualization of the PSC permitted to identify the origin of bleeding, and the coagulation of the $\mathrm{PhA}$ solved the epistaxis. To the best of our knowledge, this is the first case of spontaneous epistaxis from the $\mathrm{PhA}$ treated surgically.

Although rare, the PhA may be an anastomotic artery between the distal IMA and the ascending pharyngeal artery ( $\mathrm{APhA}$ ) [or even rarer with the accessory meningeal artery (AMA), or the ascending palatine artery] [4-7]. This anastomosis takes place in the region of the Eustachian tube, where both the PhA and APhA supply, and the blood is carried in a retrograde fashion from the APhA to the IMA

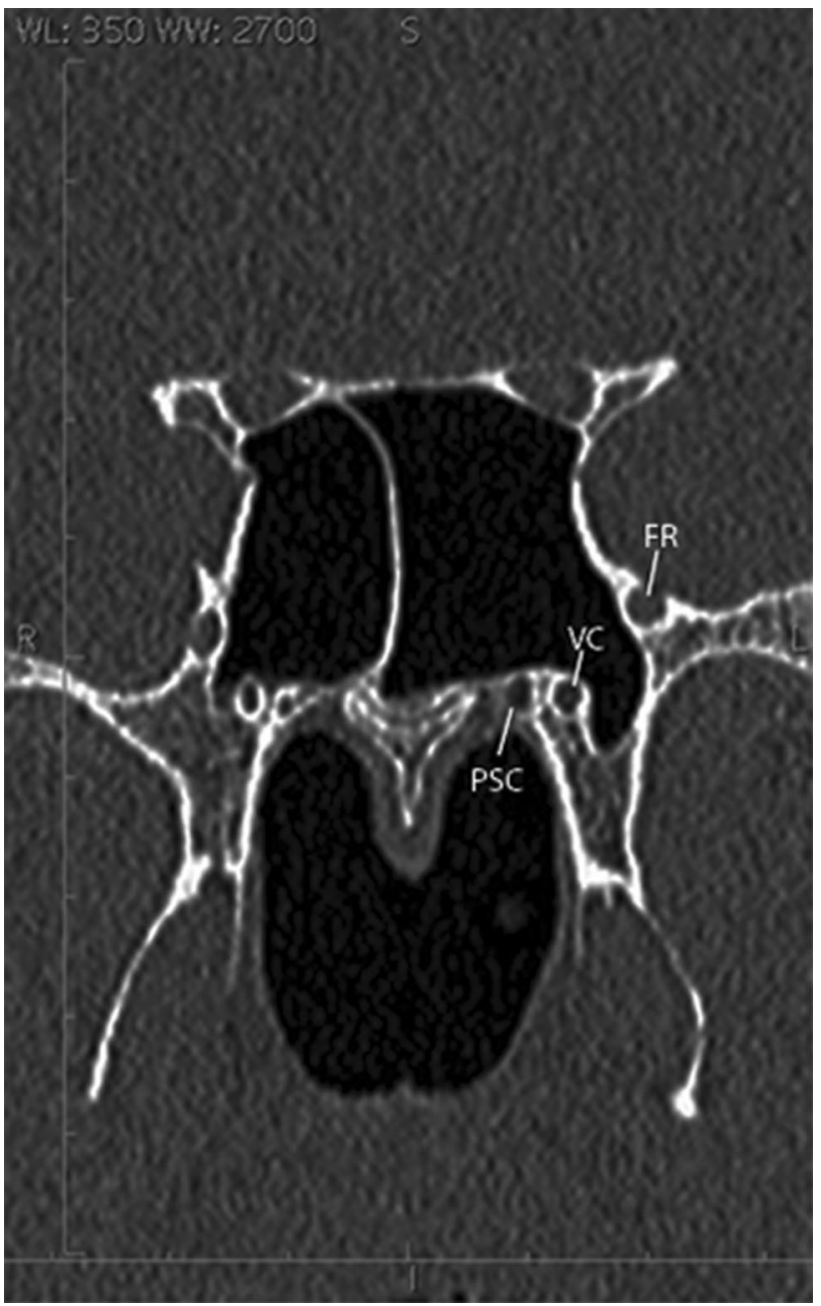

Fig. 5 Coronal CT scan showing the relationship between palatosphenoidal canal (PSC), vidian canal (VC) and foramen rotundum (FR). The PSC is the most medial one, sited at the level of the basisphenoid. The FR is at the most lateral position and communicates with the middle cranial fossa. Between these two foramens, the VC can be identified at the base of the pterygoid

through the PhA [6, 7]. Hacein-Bey et al. [4] described this condition in a case treated initially with ipsilateral IMA clipping for a recurrent posterior epistaxis. This procedure failed and a secondary angiography recognized a pseudoaneurism of the SPA immediately distal to the surgical clips which had remained patent mainly through an anastomotic flow from the ipsilateral APhA via the PhA. This was the source of the recanalization of the clipped IMA with a reconstituted blood flow. The epistaxis was finally resolved by superselective embolization of the involved branches.

The PSC can be identified on routine coronal CT and MR images and this may play a role in evaluating patients with intractable epistaxis. Rumboldt et al. [7] in their radiological study proposed that a wide PSC seen on CT, in patients with recurrent epistaxis, could raise suspicion for 


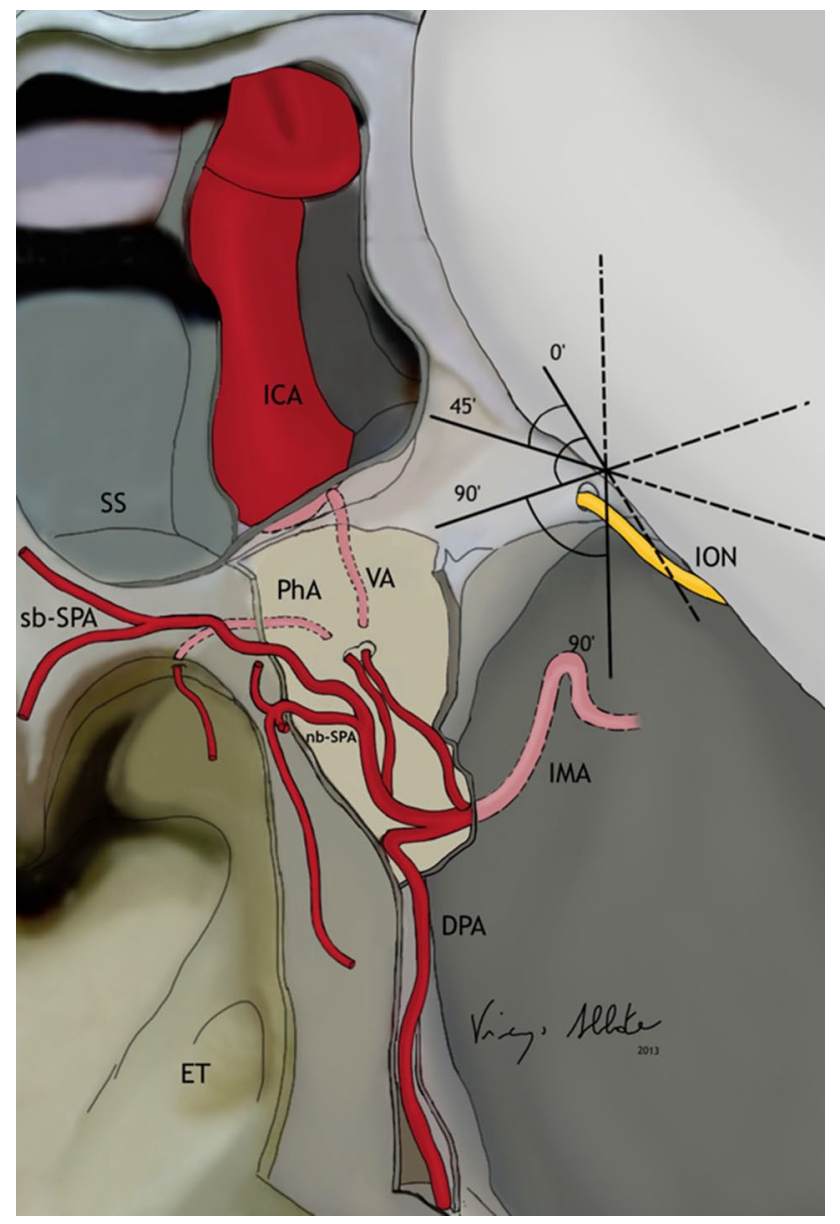

Fig. 6 Schematic illustration of the topographical relationship of the distal branches of the left internal maxillary artery (IMA) in a frontal view through the nose. The sphenopalatine artery (SPA) and its branches are placed horizontally in a coronal plane. Vertically, the descending palatine artery (DPA) is entering the greater palatine foramen. Going posteriorly to the SPA and sphenopalatine foramen, the pharyngeal artery $(\mathrm{PhA})$ has an anticlockwise oblique $45^{\circ}$ direction in the coronal plane, going from the pterygopalatine fossa to the nasopharynx. Laterally and slightly superiorly to the $\mathrm{PhA}$, the vidian artery (VA) has an orthogonal direction in relation to the SPA and is directed towards the internal carotid artery (ICA) and foramen lacerum. ET eustachian tube, ION infraorbital nerve, $n b-S P A$ nasal branches of the sphenopalatine artery, $s b-S P A$ septal branches of the sphenopalatine artery, $S S$ sphenoid sinus

the site of origin. Knowing the macroscopic and radiologic anatomy of the PSC is important for all otolaryngologists, interventional radiologists and neuroradiologists, in the preoperative assessment of the patients, to avoid considering the PSC, as an unreal variation of the vidian canal ("vidian canal duplication") [7] (Fig. 5).

Despite an increased volume of literature describing endoscopic skull base anatomy, the PSC, its anatomic relationships, and its importance in endoscopic endonasal transpterygoid approaches have not been studied in depth. The importance of the vidian canal as a landmark for the identification of the internal carotid artery (ICA) has been discussed exhaustively in the literature in the last few years [8]. However, during the identification of the vidian canal, the PSC can be confused by surgeons as to be the vidian canal in the beginning of their experience. Understanding of the topography and trajectory of these canals is of outmost importance to identify safely the vidian canal and preserve the vidian artery and nerve when necessary [2]. During endoscopic endonasal dissection of the PPF, it is important to understand the topographical relationship of the distal branches of the IMA. Approaching the PPF, the first artery to be identified in a coronal plane is the SPA and its branches (for the turbinates and septum). Vertically to the SPA, the DPA entering the greater palatine foramen is found. Going posteriorly to the SPA and sphenopalatine foramen, the second branch to be identified is the $\mathrm{PhA}$, which has an oblique or horizontal direction in the coronal plane, going from the PPF to the nasopharynx with an anticlockwise direction on the left side and a clockwise one on the right side. The third artery identified laterally and slightly superiorly to the $\mathrm{PhA}$ is the vidian artery which has an orthogonal direction in relation to the SPA (Fig. 6). As for vidian artery and nerve preservation, the same concept of PhA and PSC identification is to be applied when it is necessary to sacrifice the vidian nerve, as in the case of vidian neurectomy for tumors arising or spreading along the nerve [9].

The PSC and PhA have an important role also in cases of nasopharyngeal tumors. During the radiological assessment of these patients, identification of these two anatomical structures may play a role in detecting the spread of the tumor from the nasopharynx to the PPF [7]. In addition, when an endoscopic nasopharyngectomy is indicated, mostly in selected recurrent and in some selected primary nasopharyngeal carcinomas, initial identification of the SPA, DPA, vidian artery and PhA permits lateral mobilization of the soft tissues in the PPF [10]. Moreover, the coagulation of the $\mathrm{PhA}$ reduces significant bleeding in the surgical field, specially when the Eustachian tube is dissected, as these branches supply the region of the tube.

Furthermore, in endoscopic skull base reconstructive procedures using the nasoseptal flap, the $\mathrm{PhA}$ has to be identified and sectioned together with the vidian artery and DPA, to release laterally the pedicle of the flap [11]. This step offers more length and mobility to the flap, which otherwise may result short and not be able to reach adequately the area of reconstruction [12].

\section{Conclusion}

In case of posterior epistaxis, the SPA is not to be considered always the point of bleeding. When coagulation of this artery does not solve the bleeding, the $\mathrm{PhA}$ although rare 
may be the site of origin of epistaxis. In these means, a CT scan may be helpful in recognizing a wide PSC. Moreover, knowing the anatomy of the PhA and PSC is of outmost importance in endoscopic transpterygoid and nasopharyngeal procedures, to identify the vidian canal, evaluate nasopharyngeal cancer spread in the PPF, reduce bleeding during surgery of the nasopharynx and harvest adequately the pedicle of the nasoseptal flap.

Conflict of interest We declare that the manuscript has been neither previously published nor under consideration elsewhere and that none of the authors has any conflict of interest, financial or otherwise.

\section{References}

1. Testut L, Latarjet A (1971) Traité d'Anatomie Humaine. Neuvième édition. G. Doin \& C.ie, Paris

2. Pinheiro-Neto CD, Fernandez-Miranda JC, Rivera-Serrano CM, Paluzzi A, Snyderman CH, Gardner PA, Sennes LU (2012) Endoscopic anatomy of the palatovaginal canal (palatosphenoidal canal): a landmark for dissection of the vidian nerve during endonasal transpterygoid approaches. Laryngoscope 122:6-12

3. Borden NM, Dungan D, Dean BL, Flom RA (1996) Posttraumatic epistaxis from injury to the pterygovaginal artery. AJNR Am J Neuroradiol 17:1148-1150

4. Hacein-Bey L, Rosenbloom JS, Pile-Spellman J, Schwarzenberger J, Ho P, Duong H, Eisenberg L, Close LG (1997) Anastomoses in recurrent epistaxis. J Vasc Interv Radiol 8:535-538
5. Duncan IC, Dos Santos C (2003) Accessory meningeal arterial supply to the posterior nasal cavity: another reason for failed endovascular treatment of epistaxis. Cardiovasc Intervent Radiol 26:488-491

6. Hacein-Bey L, Daniels DL, Ulmer JL, Mark LP, Smith MM, Strottmann JM, Brown D, Meyer GA, Wackym PA (2002) The ascending pharyngeal artery: branches, anastomoses, and clinical significance. AJNR Am J Neuroradiol 23:1246-1256

7. Rumboldt Z, Castillo M, Smith JK (2002) The palatovaginal canal: can it be identified on routine CT and MR imaging? AJR Am J Roentgenol 179:267-272

8. Vescan AD, Snyderman CH, Carrau RL, Mintz A, Gardner P, Branstetter B 4th, Kassam AB (2007) Vidian canal: analysis and relationship to the internal carotid artery. Laryngoscope 117:1338-1342

9. Osawa S, Rhoton AL Jr, Seker A, Shimizu S, Fujii K, Kassam AB (2009) Microsurgical and endoscopic anatomy of the vidian canal. Neurosurgery 64(5 Suppl 2):385-411 (discussion 411-412)

10. Castelnuovo P, Nicolai P, Turri-Zanoni M, Battaglia P, Bolzoni Villaret A, Gallo S, Bignami M, Dallan I (2013) Endoscopic endonasal nasopharyngectomy in selected cancers. Otolaryngol Head Neck Surg 149:424-430

11. Karligkiotis A, Meloni F, Herman P, Castelnuovo P (2014) How to avoid mucocele formation under pedicled nasoseptal flap. Am J Otolaryngol (in press)

12. Terranova P, Karligkiotis A, Gallo S, Meloni F, Bignami M, Castelnuovo P (2013) A novel endoscopic technique for long term patency of cholesterol granulomas of the petrous apex. Laryngoscope 123:2639-2642 